\title{
A study on the efficacy and safety of combining dental surgery with tonsillectomy in pediatrics
}

This article was published in the following Dove Press journal:

Clinical, Cosmetic and Investigational Dentistry

\section{Faizaan Syed' \\ Joshua C Uffman ${ }^{1,2}$ \\ Dmitry Tumin' \\ Catherine M Flaitz ${ }^{3,4}$ \\ Joseph D Tobias ${ }^{1,2}$ \\ Vidya T Raman ${ }^{1,2}$}

'Department of Anesthesiology \& Pain Medicine, Nationwide

Children's Hospital, ${ }^{2}$ Department of

Anesthesiology \& Pain Medicine, The

Ohio State University College of

Medicine, ${ }^{3}$ Department of Dentistry,

Nationwide Children's Hospital,

${ }^{4}$ Division of Dentistry, The Ohio

State University College of Dentistry,

Columbus, $\mathrm{OH}$, USA
Correspondence: Faizaan Syed

Department of Anesthesiology \&

Pain Medicine, Nationwide Children's

Hospital, 700 Children's Drive,

Columbus, $\mathrm{OH} 43205$, USA

$\mathrm{Tel}+\mathrm{I} 6147224096$

Fax +l 6147224203

Email Faizaan.Syed@Nationwidechildrens. org
Purpose: Few data exist on combining pediatric surgical procedures under a single general anesthetic encounter (general anesthesia). We compared perioperative outcomes of combining dental surgical procedures with tonsillectomy during one anesthetic vs separate encounters.

Methods: We classified elective tonsillectomy \pm adenoidectomy and restorative dentistry as combined (group C) or separate (group S). Outcomes included anesthesia time, recovery duration, the need for overnight hospital stay, and postoperative complications.

Results: Patients aged $4 \pm 1$ years underwent tonsillectomy and dental surgery in combination $(n=7)$ or separately $(n=27)$. No differences were noted in total anesthesia time (C: median: 150 , interquartile range [IQR]: 99, 165 vs S: median: 109 , IQR: 92, 132; 95\% CI of difference in median: $-58,+10$ minutes; $P=0.115$ ) and total recovery time (C: median: 54 , IQR: 40,108 vs S: median: 72 , IQR: 58,$109 ; 95 \%$ CI of difference in median: $-16,+48$ minutes; $P=0.307)$. The need for overnight stay (C: 4 of $7, \mathrm{~S}: 20$ of $27 ; P=0.394)$ did not differ between the groups. No postoperative complications were noted in either group.

Conclusion: These preliminary data support the potential feasibility of combining dental procedures with tonsillectomy during a single anesthetic encounter. Such care may not only reduce costs but also limit parental work absences and increase convenience for patient families. When compared with procedures performed separately, combined procedures did not result in increased morbidity or significant changes in postoperative outcomes.

Keywords: combined, separate, tonsillectomy, adenoidectomy, dental, surgery, anesthesia

\section{Introduction}

Children frequently require anesthetic care for minor surgical procedures such as dental rehabilitation and tonsillectomy. While generally safe and effective with a limited risk of morbidity and mortality, ${ }^{1}$ the performance of minor surgical procedures under separate anesthetics impacts family and societal issues including days missed from work and school. Combining multiple procedures in a single administration of general anesthesia (GA) is an attractive option for reducing cost and length of hospital stay for separate procedures and increasing patient/family convenience. ${ }^{2-4}$ Currently, evidence suggests that combined surgical procedures are feasible and generally safe. However, evaluating perioperative outcomes of these combined procedures has introduced significant methodological challenges..$^{2-4}$ In prior analyses of combined surgical procedures, comparisons were made to patients undergoing single procedure under $\mathrm{GA}^{4}$ or to institutional averages of operative time, cost, and other characteristics of a particular procedure. $^{3}$ These comparisons have incurred unavoidable bias by drawing 
reference values from a group of patients who may only have required one of the combined procedures and may have been different from the group of patients requiring multiple procedures. In this study, we piloted a method of comparing combined procedures with a cohort of patients undergoing multiple procedures under separate anesthetic encounters during a similar time period. Our hypothesis was that combining two procedures under a separate anesthetic, although challenging given the need to coordinate different services, would not result in increased perioperative complications or impact outcomes.

\section{Methods}

The study was approved by the institutional review board (IRB) of Nationwide Children's Hospital with a waiver of individual consent (IRB16-00457). As a retrospective chart review, the IRB does not request informed consent for retrospective studies. This is a standard practice as no randomization occurred, and there was no change in clinical care. Study data were stored and handled securely to protect confidentiality, and only the research personnel involved had access to it. All elective or ambulatory surgical procedures under GA in children aged $\leq 18$ years at our institution were identified over a 12-month time period. Based on an initial review of the most frequent procedures performed individually and in combination, patients were selected for analysis if they had undergone tonsillectomy \pm adenoidectomy (TA) and comprehensive restorative dentistry (CRD) during the study period. Patients undergoing any other procedures under GA were excluded, so that the only surgical procedures performed under GA in the study cohort were TA and CRD. These patients were then classified according to whether their procedures were combined (group C) or separate (group S). Separate procedures in the comparison group were included only if both separate procedures were performed in the main OR. Obesity was defined as body mass index (BMI)-for-age equaling or exceeding the 95th percentile, as established by the Centers for Disease Control and Prevention for children aged 2 years and older.

The primary outcome was total anesthesia time. Secondary outcomes included total time in the postanesthesia care unit (PACU; in group S, this included the sum of both PACU stays), the need for a prolonged PACU stay ( $>60$ minutes, after one or both procedures), and whether patients required overnight hospital stay (in group S, after one or both procedures). Additional data extracted from the medical record included patient demographic characteristics, BMI, and
American Society of Anesthesiologists (ASA) status. Patient records were further reviewed to determine if any postoperative complications had occurred. Continuous data were summarized as mean with standard deviation, and categorical data were summarized as counts with percentage. In group S, measures of age and BMI were averaged between procedures and the higher of the two ASA status scores was reported. Median with interquartile range (IQR) for continuous data was compared by a Mann-Whitney $U$-test, while categorical characteristics were compared between groups using Fisher's exact test. Finally, the feasibility of combining TA and CRD for patients in group $\mathrm{S}$ was determined according to referral dates (ie, whether the later procedure had been scheduled before completion of the earlier procedure). Data analysis was performed in Stata/IC 13.1 (StataCorp LP, College Station, TX, USA), and two-tailed $P<0.05$ was considered statistically significant.

\section{Results}

The study cohort included 34 patients ( 16 boys and 18 girls) aged 2-8 years (mean age $4 \pm 1$ years) who had undergone both TA and CRD, either during separate or a single combined GA, during the 12-month study period. Twenty-seven patients had procedures performed separately (group S), while seven patients had these procedures combined under a single anesthetic (group C; Table 1). Among the 27 patients in group S, average time between procedures was $145 \pm 86$ days (range 28-317 days). Review of referral dates for the two separate procedures determined that only three of these patients could have feasibly undergone combined TA and CRD.

Patients in groups $\mathrm{S}$ and $\mathrm{C}$ did not differ in age, gender composition, or highest recorded ASA status (Table 1). No difference was noted in total anesthesia time (C: median: 150, IQR: 99, 165 vs S: median: 109, IQR: 92, 132; 95\% CI of difference in median: $-58,+10$ minutes; $P=0.115)$ and total PACU recovery time (C: median: 54 , IQR: 40,108 vs S: median: 72, IQR: 58, 109; 95\% CI of difference in median: $-16,+48$ minutes; $P=0.307)$. When considering the prolonged PACU stay of $>60$ minutes as a dichotomous outcome, there was stronger evidence for an advantage of combining procedures, with only three of the seven patients in group $C$ requiring PACU stay $>60$ minutes, compared to 22 of the 27 patients in group $\mathrm{S}$ requiring prolonged PACU stay after one or both procedures $(P=0.061)$. However, the need for overnight stay did not differ between the two groups $(\mathrm{C}: 4$ of 7 vs S: 20 of 27; $P=0.394$ ). No postoperative complications were noted among any of the patients in either group. 
Table I Characteristics of patients undergoing combined vs separate tonsillectomy and dental surgery procedures

\begin{tabular}{|c|c|c|c|}
\hline \multirow[t]{2}{*}{ Variable } & \multirow{2}{*}{$\begin{array}{l}\text { Group S: separate procedures }(n=27) \\
\text { Median (IQR) or } n(\%)\end{array}$} & \multirow{2}{*}{$\begin{array}{l}\text { Group C: combined procedure }(n=7) \\
\text { Median (IQR) or } n(\%)\end{array}$} & \multirow[t]{2}{*}{$P$-value } \\
\hline & & & \\
\hline Age (years) ${ }^{b}$ & $4(3,6)$ & $4(3,5)$ & 0.604 \\
\hline Gender (male/female) & $13 / 14$ & $3 / 4$ & $>0.999$ \\
\hline ASA status $^{c}$ & & & $>0.999$ \\
\hline 1 & I (4) & 0 & \\
\hline 2 & $21(78)$ & $6(86)$ & \\
\hline 3 & $5(19)$ & I (14) & \\
\hline BMI $\left(\mathrm{kg} / \mathrm{m}^{2}\right)^{\mathrm{b}}$ & $17(15,18)$ & $16(16,18)$ & 0.815 \\
\hline Obesity $^{d}$ & $6(22)$ & $I(14)$ & $>0.999$ \\
\hline Total anesthesia time (minutes) & $109(92,132)$ & $150(99,165)$ & 0.115 \\
\hline Total PACU time (minutes) & $72(58,109)$ & $54(40,108)$ & 0.307 \\
\hline Prolonged PACU staye & $22(8 I)$ & $3(43)$ & 0.061 \\
\hline Overnight stay requirede & $20(74)$ & $4(57)$ & 0.394 \\
\hline Asthma & $3(11)$ & 0 & $>0.999$ \\
\hline Seizure disorders & I (4) & 0 & $>0.999$ \\
\hline Developmental delay & $8(30)$ & I (14) & 0.160 \\
\hline Cerebral palsy & I (4) & 0 & $>0.999$ \\
\hline
\end{tabular}

Notes: aFisher's exact test for categorical variables and Mann-Whitney U-test for continuous variables. ${ }^{b}$ Average of encounters, for patients undergoing separate procedures. 'Maximum of encounters, for patients undergoing separate procedures. ${ }^{\mathrm{B} M M I-f o r-a g e ~ e q u a l i n g ~ o r ~ e x c e e d i n g ~ t h e ~} 95$ th percentile. ${ }^{\mathrm{A}} \mathrm{After}$ either surgical procedure ( $>60$ minutes), for patients undergoing separate procedures.

Abbreviations: ASA, American Society of Anesthesiologists; BMI, body mass index; IQR, interquartile range; PACU, postanesthesia care unit.

\section{Discussion}

Combined procedures have been considered as a costeffective strategy to reduce the need for repeated hospitalization, missed days at school, missed work days for parents, and repeated exposure to anesthetic care. ${ }^{2-4}$ However, the concern remains that the prolongation of anesthetic care to allow combined procedures might be associated with an increased incidence of postoperative complications. Our preliminary data support the safety and efficacy of combining minor surgical procedures under the same anesthetic. We found that patients undergoing combined procedures, when compared with patients undergoing the same procedures under separate anesthetics, had no difference in postoperative complications, the need for postoperative overnight admission, but did document a decreased incidence of a prolonged PACU stay. American Academy of Pediatrics (AAP) responded to a recent Food and Drug Administration warning regarding the use of repeated or prolonged anesthetic administration (procedures lasting $>3$ hours) in children younger than 3 years. ${ }^{5}$ AAP stated that recent controlled trials in humans showed no adverse developmental concerns in short anesthetics. ${ }^{5}$ This further supports our study in stating the need for combined procedures to reduce anesthesia time and repeated anesthetic exposures.

In a previous study evaluating the combination of surgical procedures across services under a single anesthetic encounter, Stapleton et $\mathrm{al}^{3}$ reported that combining dental and other procedures resulted in reduced hospital and recovery time (312 vs 133 minutes) and significant financial savings. However, these authors noted certain challenges posed by combining these procedures, related to scheduling complexity, presurgical planning, coordination with insurance companies, and the process of establishing whether it is appropriate to combine procedures. ${ }^{3}$ In a similar retrospective study in adults, the total duration of surgery, anesthesia time, and hospital stay were lower for combined procedures than staged procedures; however, the authors expressed concern that one procedure might adversely influence the outcome of the other in a combined case or that patient discomfort may be greater when combining surgical procedures. ${ }^{2} \mathrm{~A}$ case-control study in adult patients, comparing combined abdominoplasty and intra-abdominal gynecologic procedures with isolated abdominoplasty controls and isolated gynecologic surgery controls, reported reductions in total operative time, estimated blood loss, and total days of hospitalization when the procedures were performed in combination. ${ }^{4}$

An inherent limitation of our study is its small cohort size; however, to eliminate biases related to study design, we chose to compare combined procedures in one cohort to another cohort, which included patients who underwent the same two procedures (dental surgery plus tonsillectomy) under separate anesthetic encounters, thereby limiting the cohort despite the large number of procedures performed at our institution each year $(>36,000)$. The preliminary data demonstrate the safety of combining these procedures as we noted no difference in recovery times, fewer patients requiring a prolonged PACU 
stay, no increased need for overnight hospitalization, and no adverse events in patients undergoing combined procedures. The high incidence of the need for postoperative admission in both groups likely relates to our standard clinical practice of routine admission for patients with obstructive sleep apnea (OSA) following tonsillectomy or adenotonsillectomy. ${ }^{6}$ The limited size of the study cohort and the short duration of the two surgical procedures studied may have led to a study that was not powered to show a statistical significance when evaluating operating times. Furthermore, to expedite operating room (OR) turnover times at our institution, tracheal extubation is frequently preformed in the PACU, thereby limiting time from the completion of the surgical procedure until exit from the OR. ${ }^{7}$

Additional factors may influence operating and anesthetic times. In the study by Balraj et al, ${ }^{2}$ although there is a comparison of patients undergoing combined procedures with patients undergoing separate procedures, all procedures are performed by a single service (otolaryngology). Therefore, improvements attributed to combined procedures in that study may not be generalizable to combinations of procedures involving multiple services, as explored in our analysis. On nonparametric analysis, there was no evidence for shorter total anesthesia time among patients undergoing combined procedures, which may have been due to factors such as going through a secondary timeout, travel, or wait time associated with a second surgeon arriving from a different operating location and preparation for the second case. Logistical challenges to combining procedures include determining which service's block time is used, whether combined procedures require one team wait on another team to finish the first procedure, and whether combined procedures require moving cases to the main OR as compared to a separate surgical center. Clear identification of these factors may provide further clarification of ways to decrease total operative times when procedures are combined. Also, severe OSA is a relative contraindication to combining procedures, but our study did not have the polysomnography data on most patients, preventing us from definitively investigating the safety of combining procedures in patients with OSA.

To accomplish the potential for combining procedures, institutional or individual practices frequently must change or adapt. In our institution, CRD and TA may be performed in combination depending on the extent and severity of the disease (sleep disordered breathing, recurrent throat infections, adenotonsillar hypertrophy, odontogenic infection, and impacted teeth), patient's cooperation during outpatient procedures, feasibility (whether the second diagnosis was made before the first procedure was completed), and whether anticipated benefits outweigh the risks of a longer surgical and anesthetic time. Such coordination is greatly facilitated by the electronic health record that includes all medical and dental specialties and central scheduling. Typically, in combined cases, our dentistry service was the initiator based on parental request to coordinate the care. The dental service would search for a previously scheduled procedure to which the required CRD procedures could be added as long as it was scheduled in a main OR, with time available and agreement of the otolaryngology surgeon. This is facilitated by the fact that the CRD procedures are generally not emergent or urgent and, therefore, may be delayed for months so that the treatment could be completed concurrently during another scheduled procedure.

The final determination of a combined procedure is made by the otolaryngology surgeon or is constrained by logistical considerations (ie, whether there is additional OR time available to perform both procedures and whether the procedures are scheduled with enough time available for proper planning to ensure surgeon availability). As illustrated in our results, this posed a significant challenge because indications for each procedure must be diagnosed within a close time frame. Combining procedures also requires coordination of departments, OR availability, and postoperative follow-up. To further evaluate the benefits and obstacles of combining cases, reasons for not combining procedures (eg, severity of disease), and logistical data on availability of surgeons, anesthesiologists, and OR, time should be investigated.

Although we included a comparable patient population undergoing identical procedures under one or two anesthetic encounters, our study is limited by some aspects of the available data. Most importantly, despite reviewing all elective procedures $>1$ year, we found very few patients undergoing combined procedures who could be compared with patients undergoing the exact same procedures under separate anesthetics during the study period. For example, the lack of evidence for a difference in total recovery times may have been related to the sample size. However, this limitation must be weighed against strategies used by past studies with more permissive criteria for constructing the control group, which may have led to bias due to heterogeneity in their comparison cohorts. Additionally, our study focused on perioperative outcomes, so we did not evaluate differences in total surgery cost. Another limitation of our study was that we did not perform a priori power calculation for this study as we had planned to analyze all patients meeting inclusion criteria. 
Despite the logistic constraints of managing two separate surgical services, our preliminary data suggest that the combination of CRD and TA under a single anesthetic is feasible and safe. Very few data exist to quantify the benefit and safety outcomes of combining two surgical procedures under one anesthetic encounter. These data are further limited by an unclear and potentially biased selection of comparison patients undergoing separate procedures. We have presented our approach to identify a valid comparison group for patients undergoing combined TA and CRD and found that patients undergoing these procedures under separate anesthetic encounters did not experience longer cumulative anesthesia time, significantly prolonged PACU time, or increased likelihood of postoperative complications including overnight hospitalization. Of importance, but not measured, select combination surgeries alleviate the impact on family and patient time such as missed days at school or work when compared with two separate surgical procedures. ${ }^{8}$ Further applications of this method to other combinations of procedures would clarify the advantages and disadvantages associated with combining pediatric surgeries that require GA.

\section{Conclusion}

With our findings, our study can be a basis for future studies that have a larger population and can help us better understand the changes combining viable procedures can bring. The design of our study is potentially useful for larger studies comparing a broader range of combined procedures. Our results could be used for power calculations when planning larger studies. A larger study could address the potential cost savings of combining cases. We can also focus more on reduction in missed school or work days when combining surgical procedures.

\section{Author contributions}

DT, CMF, JDT, and VTR contributed to the study design. FS and VTR contributed to the data collection. FS and DT contributed to the data analysis. JCU, JDT, and VTR interpreted the results. FS and DT drafted the article. CMF, JCU, JDT, and VTR critically revised the article. All authors contributed toward data analysis, drafting and revising the paper and agree to be accountable for all aspects of the work.

\section{Disclosure}

The authors report no conflicts of interest in this work.

\section{References}

1. van der Griend BF, Lister NA, McKenzie IM, et al. Postoperative mortality in children after 101,885 anesthetics at a tertiary pediatric hospital. Anesth Analg. 2011;112(6):1440-1447.

2. Balraj A, Kurien M, Job A. Concurrent surgeries in ENT: a cost-effective and safe approach in patients requiring multiple definitive procedures. J Laryngol Otol. 2004;118(1):31-33.

3. Stapleton M, Sheller B, Williams BJ, Mancl L. Combining procedures under general anesthesia. Pediatr Dent. 2007;29(5):397-402.

4. Sinno S, Shah S, Kenton K, et al. Assessing the safety and efficacy of combined abdominoplasty and gynecologic surgery. Ann Plast Surg. 2011;67(3): 272-274.

5. Aappublications.org [webpage on the Internet]. AAP Responds to FDA Warning on Anesthesia Use in Children; AAP news; 2017. Available from: http://www.aappublications.org/news/2017/01/10/Anesthesia011017. Accessed February 1, 2018.

6. Raman VT, Jatana KR, Elmaraghy CA, Tobias JD. Guidelines to decrease unanticipated hospital admission following adenotonsillectomy in the pediatric population. Int J Pediatr Otorhinolaryngol. 2014;78(1): 19-22.

7. Kako H, Corridore M, Seo S, Elmaraghy C, Lind M, Tobias JD. Tracheal extubation practices following adenotonsillectomy in children: effects on operating room efficiency between two institutions. Paediatr Anaesth. 2017;27(6):591-595.

8. Stevens ML, Hubert BC, Wenzel FJ. Combined gynecologic surgical procedures and cholecystectomy. Am J Obstet Gynecol. 1984;149(3): $350-354$.
Clinical, Cosmetic and Investigational Dentistry

\section{Publish your work in this journal}

Clinical, Cosmetic and Investigational Dentistry is an international, peer-reviewed, open access, online journal focusing on the latest clinical and experimental research in dentistry with specific emphasis on cosmetic interventions. Innovative developments in dental materials, techniques and devices that improve outcomes and patient satisfac-

\section{Dovepress}

tion and preference will be highlighted. The manuscript management system is completely online and includes a very quick and fair peerreview system, which is all easy to use. Visit http://www.dovepress. $\mathrm{com} /$ testimonials.php to read real quotes from published authors. 